\title{
Normal Response of Active GLP-1 like Substances Level to Test Meal in Non-Obese Type 2 Diabetic Japanese Patients with Complications and Receiving Treatments
}

\author{
Kyuzi Kamoi ${ }^{1,4}$, Nobumasa Ohara ${ }^{2,3}$, Ikarashi Tomoo ${ }^{2,3,5}$, You Shinozaki ${ }^{1,2}$, Kazuo Furukawa ${ }^{1,2}$ and Hideo Sasaki \\ ${ }^{1}$ Center of Diabetes and Endocrine \& Metabolism Disease, Nagaoka Red Cross Hospital, Nagaoka, Niigata 940-2085, Japan \\ Department of Endocrinology and Metabolism, Niigata University, Niigata, Niigata 951-8510, Japan \\ ${ }^{3}$ Diabetes Center, Niigata Medical Center, Niigata, Niigata 950-2022, Japan \\ ${ }^{4}$ Department of Health and Nutrition, University of Niigata Prefecture, Niigata, Niigata 950-8680, Japan \\ ${ }^{5}$ Diabetes Center, Kariwa Gun Hospital, Kashiwazaki, Niigata 945-8535, Japan
}

\begin{abstract}
Background: Study has shown no significant differences in basal and postprandial plasma active glucagon-like peptide-1 ( $p$-active GLP-1) levels following test meal (TM) between complication- and treatment-naïve non-obese Japanese patients with type 2 diabetes (T2DM) and controls.

Methods: In non-obese Japanese patients with T2DM ( $n=23$, group 1$)$ and healthy individuals as control $(n=13$, group 2), blood levels of plasma glucose (PG), serum insulin (s-IRI), serum C-peptide (s-CPR) and p-active GLP-1 like substances (p-active GLP-1-S) were measured 0, 30, and 60 min after TM (520-560 kcal. $23 \%$ fat, $60 \%$ carbohydrate and $17 \%$ protein). $\mathrm{HbA} 1 \mathrm{c}$ levels were also measured in the groups. Patients with mean of 9.2 years disease had various complications and treatment with diet, exercise and/or oral medical drugs except incretin-related drugs for hyperglycemia.
\end{abstract}

Results: There was no significant difference in mean of sex, age, or BMl between groups. Means of HbA1c and basal and postprandial PG with area under curve (AUC), and HOMA-R were significantly higher in group 1 than in group 2. Means of HOMA- $\beta$ and insulinogenic index after ingestion of TM were significantly lower in group 1 than in group 2. However, there were no significant differences in means of basal and postprandial with AUC levels of s-IRI, $\mathrm{s}-\mathrm{CPR}$ and $\mathrm{p}$-active GLP-1-S levels between groups.

Conclusion: These results indicated that a response of p-active GLP-1-S to TM in non-obese Japanese patients with T2DM associated with a long duration of disease, various complications and various treatments with except incretin-related drugs was similar to those in non-obese healthy individuals.

Keywords: Active GLP-1; Test meal; BMI; Non-obesity; Type 2 DM

\section{Background}

Glucagon-like peptide-1 (GLP-1) and glucose-dependent insulinotropic polypeptide (GIP) are secreted in response to ingestion of nutrients. In the circulation, GLP-1 and GIP are rapidly inactivated by dipeptidyl peptidase-IV (DPP-IV), which cleaves off two $\mathrm{N}$-terminal amino acids [1].

Vilsbøll et al. reported that a low response in plasma active GLP1 (p-active GLP-1) to ingestion of breakfast test meal (TM) was seen in European patients with type 2 diabetes mellitus (T2DM) [2], while Lee et al. reported that there was no significant difference in a response of p-active GLP-1 levels following TM in complication- and treatment-naïve Japanese patients with T2DM [3]. Our preliminary study supported the findings of Lee et al. by the facts that postprandial p-active GLP-1 like substances levels following TM were not decreased in complication- and drug-non-naïve non-obese Japanese patients with T2DM (unpublished data).

To clarify a response of active GLP-1 levels in duration of disease-, complication- and treatment-non-naïve Japanese patients with T2DM, we reexamined the effects of a TM similar to the one used by Vilsbøll et al. [2] on basal and postprandial levels of p-active GLP-1 like substances ( $\mathrm{p}$-active GLP-1-S) after TM in non-obese Japanese patients with T2DM, who had a long duration of disease and various kinds of complications, and received various kinds of drugs except incretinrelated for diseases.

\section{Methods}

\section{Subjects}

Non-obese (BMI $<25.0 \mathrm{~kg} / \mathrm{m}^{2}$ ) Japanese patients with T2DM $(\mathrm{n}=23$, group 1$)$ and non-obese Japanese healthy subjects with normal glucose tolerance (NGT) as control ( $n=13$, group 2) (Table 1) were studied, in which the definition of non-obesity was based on the criteria of Japan Society for the Study of Obesity [4]. Patients with obesity and insulin treatment were excluded from this study, because that such factor may influence the p-active GLP-1 levels [5]. Groups 1 and 2 were matched by sex, age, and BMI. Demographic characteristics of the participants are presented in Table 1. Diabetic patients were diagnosed by the World Health Organization criteria [6] with $\geqq 6.5 \%$ of HbAlc

*Corresponding author: Kyuzi Kamoi, MD, M.Sc., Department of Health and Nutrition, University of Niigata Prefecture, 471, Ebigase, Higashi-ku, Niigata Niigata 950-8680, Japan, Tel: +81 025-270-1300; Fax: +81 025-270-5173 E-mail:kkam-int@echigo.ne.jp

Received September 08, 2011; Accepted October 15, 2011; Published Octobe 20, 2011

Citation: Kamoi K, Ohara N, Tomoo I, Shinozaki Y, Furukawa K (2011) Norma Response of Active GLP-1 like Substances Level to Test Meal in Non-Obese Type 2 Diabetic Japanese Patients with Complications and Receiving Treatments. J Diabetes Metab 2:147. doi:10.4172/2155-6156.1000147

Copyright: (c) 2011 Kamoi K, et al. This is an open-access article distributed unde the terms of the Creative Commons Attribution License, which permits unrestricted use, distribution, and reproduction in any medium, provided the original author and source are credited. 
Citation: Kamoi K, Ohara N, Tomoo I, Shinozaki Y, Furukawa K (2011) Normal Response of Active GLP-1 like Substances Level to Test Meal in Non-Obese Type 2 Diabetic Japanese Patients with Complications and Receiving Treatments. J Diabetes Metab 2:147. doi:10.4172/21556156.1000147

(NGSP). All patients had T2DM with around 9 years of disease from discovery (Table 1). Therefore, some had retinopathy, nephropathy or peripheral neuropathy, and asymptomatic coronary heart disease or asymptomatic cerebral vascular diseases. The occurrences of retinopathy and nephropathy were examined by ophthalmologist and by mean of albuminuria. To diagnose diabetic peripheral neuropathy, the patient's history of symptoms for the foot and ankle was obtained and performed simple in-office tests on the feet and legs. This evaluation included assessment of the patient's reflexes, light touch and vibration. In some cases, additional neurologic tests were performed, respectively. The macrovascular complications were defined by past medical history. They were treated with diet, exercise and/or oral medical drugs for hyperglycemia. Oral medical drugs were consisted of $a$-glucosidase inhibitors ( $\alpha-\mathrm{GI})$, sulfonylurea (SU), biguanide (BG), thiazolidine (TZD) or combinations with them (Table 1). Some patients were treated with anti-hyperlipidemic or anti-hypertensive drugs.

Non-obese control subjects were recruited from persons with NGT for a $75 \mathrm{gOGTT}$ with $<6.5 \%$ of HbAlc (NGSP). None of the participants had a history of gastrointestinal disease, anemia, or impaired liver function and none were receiving any other medications.

Written informed consent was obtained from all subjects after informing them of purpose and nature of the study. This study was performed in accordance with the Declaration of Helsinki and with the approval of our hospital ethics committees.

\section{Study design}

The study design was previously reported [5]. After 10-hours overnight fast, subjects were studied at 9:00 a.m. The TM (520-560 $\mathrm{kcal}$ ) comprised of $23 \%$ fat, $60 \%$ carbohydrate and $17 \%$ protein [5]. Patients stopped all medications during the study.

Insulin resistance and beta-cell function were assessed using HOMA-R and HOMA- $\beta$ (\%), which were calculated by the equations reported previously $[3,5,7]$. Insulinogenic index was also calculated $[3,5]$.

Blood samples were collected in ice-cooled tubes from the inserted cannula immediately before, and 30 and 60 minutes after ingestion of the TM and were separated by centrifugation at $4^{\circ} \mathrm{C}$ for later determination of PG, s-IRI, serum immunoreactive C-peptide (s-CPR) and p-active GLP-1-S levels. The sample collected was also used to measure HbA1c levels. They for active GLP-1-S and glucose were collected in ice-cooled vacutainers containing EDTA with $10 \mu \mathrm{LPP}$ IV inhibitor (diprotin) per $\mathrm{mL}$ of blood and in vacutainers containing $\mathrm{NaF}$, respectively [5].

Increment levels of PG, s-IRI, s-CPR and p-active GLP-1-S after ingestion of the TM as AUC were calculated as method reported previously [5].

\section{Assay methods}

All assay methods were same as previous reports [5]. Briefly, HbAlc was measured using high-performance liquid chromatography. The value was expressed as NGSP equivalence. PG was measured by using oxidase method. S-IRI and s-CPR were measured by two-site sandwich immunoassay kits [5]. The intra- and inter-assay coefficients of variation are both $<5 \%$.

P-active GLP-1 was measured in the unextracted sample by twosite sandwich enzyme immunoassay using a commercially available ELISA active GLP-1 kit (Linco Research, St. Charles, MO, USA) at SRL, Inc. (Tokyo, Japan) as previously reported method [5]. As foreign substances in unextracted samples might interfere in the assay $[7,8]$, we represented that the value measured by this kit as p-active GLP-1-S. The antibody provided with kit specifically recognizes the $\mathrm{N}$-terminal region of active GLP-1 (7-36 and 7-37), but not other forms of GLP-1 (1-36, 1-37, 9-36 and 9-37). The limit of detection for this assay is $<2.0$ $\mathrm{pmol} / \mathrm{L}$. The intra- and inter-assay coefficients of variation were both $<13 \%$ [5].

\section{Statistical methods}

Results are expressed as means \pm SEM. Differences between means of basal variables, insulinogenic index or AUC in groups were evaluated statistically by chi square or unpaired $t$ tests with or without Welch's correction $[3,5]$.

The same method reported previously [5] was used to determine how the response was affected by TM in groups. Two-tailed $p$-values $<$ 0.05 were considered as statistically significant. Analysis was performed using GraphPad Prism version 5.04 (GraphPad Software, La Jolla, CA, USA).

\section{Results}

\section{Basal levels}

There was no significant difference in mean of sex, age or BMI between groups. Means of HbA1c and PG, and HOMA-R were significantly higher in group 1 than in group 2, whereas mean of

\begin{tabular}{|l|l|l|l|}
\hline & $\begin{array}{l}\text { Type 2 diabetic } \\
\text { patients }\end{array}$ & Control & p value \\
\hline Number & 23 & 13 & \\
\hline Age & $58 \pm 2.7$ & $52 \pm 2$ & 0.975 \\
\hline Gender (males/females) & $18 / 5$ & $5 / 8$ & 0.169 \\
\hline BMI (kg/m $\left.{ }^{2}\right)$ & $21 \pm 0.3$ & $21 \pm 0.5$ & 1.000 \\
\hline HbA1c (\%) (NGDP) & $9.1 \pm 0.30$ & $5.4 \pm 0.01$ & $<0.001$ \\
\hline Plasma glucose (mg/dL) & $151 \pm 9$ & $92 \pm 2$ & $<0.001$ \\
\hline Serum immunoreactive insulin ( $\mu \mathrm{U} / \mathrm{mL})$ & $5.2 \pm 0.6$ & $3.7 \pm 0.6$ & 0.163 \\
\hline HOMA-R & $1.9 \pm 0.2$ & $0.8 \pm 0.1$ & 0.001 \\
\hline HOMA- $\beta$ (\%) & $25.1 \pm 3.4$ & $80.1 \pm 5.1$ & 0.001 \\
\hline Serum immunoreactive C-peptide (ng/mL) & $1.7 \pm 0.1$ & $1.4 \pm 0.1$ & 0.073 \\
\hline $\begin{array}{l}\text { Plasma immunoreactive active GLP-1 like } \\
\text { substance (pmol/L) }\end{array}$ & $4.0 \pm 0.5$ & $2.6 \pm 0.5$ & 0.063 \\
\hline Duration of diabetes from discovery (year) & $9.2 \pm 1.5$ & & \\
\hline Retinopathy (NDR/SDR//PPDR/PDR) & $19 / 1 / 0 / 3$ & & \\
\hline Peripheral neuropathy & 8 & & \\
\hline Nephropathy (Normo/Micro/Macro) & $17 / 5 / 1$ & & \\
\hline Macroangiopathy (asymptomatic) & 3 & & \\
\hline Diabetic treatment (a-GI/BG/TZD/SU) & $1 / 15 / 12 / 12$ & & \\
\hline Dyslipidemia treatment & 2 & & \\
\hline Hypertension treatment & 5 & & \\
\hline
\end{tabular}

Table 1: Clinical characteristics of complications- and treatment-non-naïve nonobese Japanese patients with type 2 diabetes mellitus and non-obese Japanese controls with normal glucose tolerance.

Data are expressed as means \pm SEM. Each value was collected in the morning with fasting. HOMA-R $=$ fasting immunoreactive insulin (IRI) level $\times$ fasting plasma glucose (PG) level /405. HOMA- $\beta$ =fasting IRI level x 360/ (fasting PG level -63 ). Differences between the means in two groups were statistically evaluated by ch square or unpaired $t$ tests with or without Welch's correction. Two-tailed values of $p<0.05$ were defined as statistically significant.

NGSP; national glycohemoglobin standard program, HOMA-R; homeostasis mode assessment- insulin resistance, HOMA- $\beta$; homeostasis model assessment- beta cell function, NDR; non-diabetic retinopathy, SDR; simple diabetic retinopathy, PPDR; pre-proliferative retinopathy, PDR; proliferative retinopathy, Normo: normoalbuminuria, Mico; microalbuminuria, Macro; macroalbuminuria, a-GI; alphaglucosidase inhibitors, BG; biguanide, TZD; thiazolizinedione, SU; sulfonylurea. 
Citation: Kamoi K, Ohara N, Tomoo I, Shinozaki Y, Furukawa K (2011) Normal Response of Active GLP-1 like Substances Level to Test Meal in Non-Obese Type 2 Diabetic Japanese Patients with Complications and Receiving Treatments. J Diabetes Metab 2:147. doi:10.4172/21556156.1000147

HOAMA- $\beta$ was significantly lower in group 1 than in group 2 . However, there was no significant difference in mean of s-IRI, s-CPR or p-active GLP-1-S levels between the groups. In group 1, mean of diabetes duration was 9.2 years, and patients had various micro- and macro-vascular complications with various degrees and received various drugs for various diseases (Table 1).

\section{Postprandial levels}

In postprandial levels after TM, mean PG at each time point was significantly higher in group 1 than in group 2, whereas mean insulinogenic index was significantly $(\mathrm{P}<0.001)$ lower in group $1(0.23$ $\pm 0.08)$ than in group $2(3.13 \pm 0.17)$. However, there were no significant differences in mean of s-IRI, s-CPR or p-active GLP-1-S at each time point between two groups, although the peak levels of p-active GLP-1-S in both groups were observed at $30 \mathrm{~min}$ after TM (Figure 1).
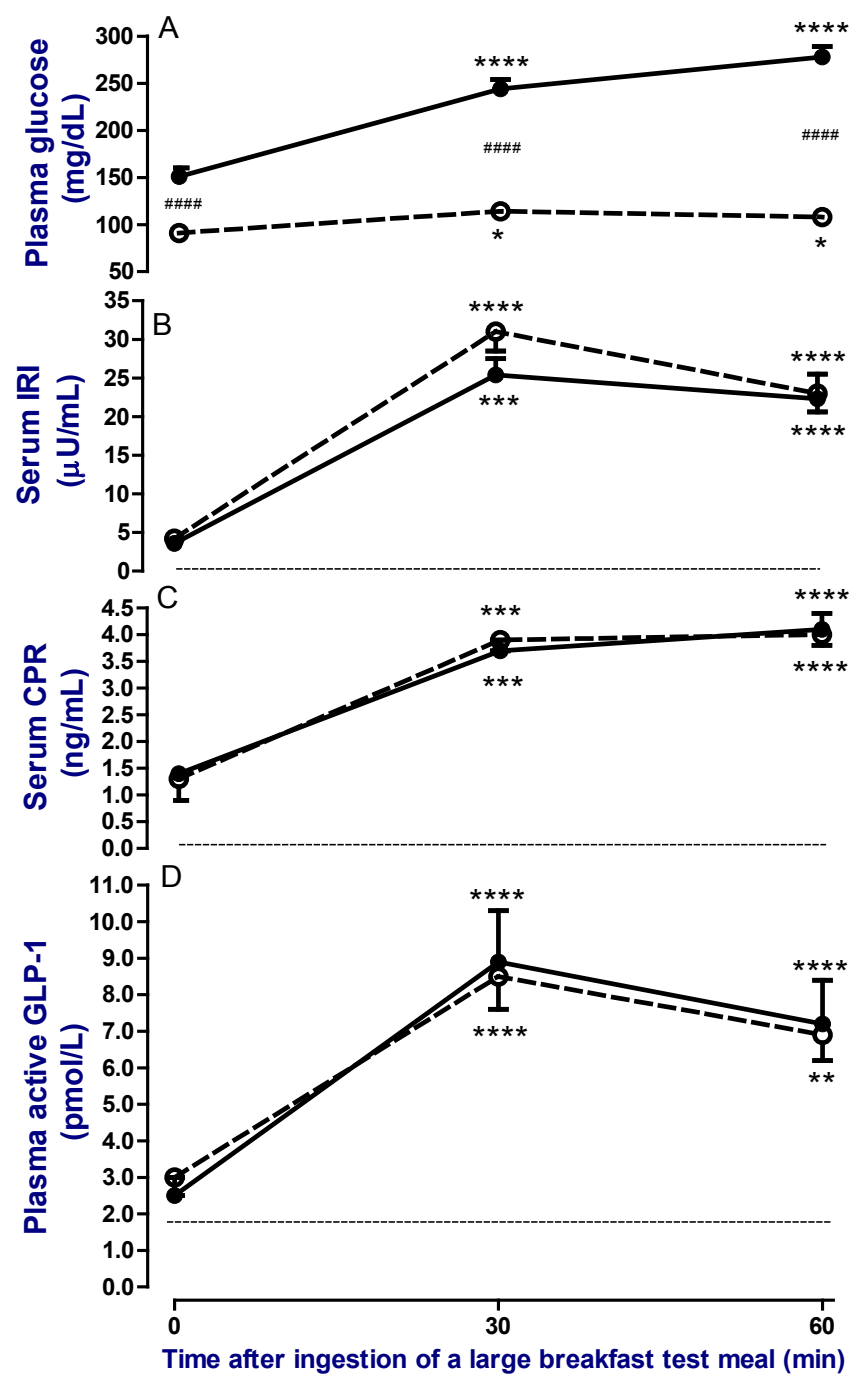

Figure 1: Change in levels of plasma glucose $(A)$, serum immunoreactive insulin (IRI) (B), serum immunoreactive C-peptide (CPR) (C) and plasma immunoreactive active GLP-1 like substances (active GLP-1 like substances) (D) before, 30 and 60 minutes after ingestion of a breakfast test meal (520$560 \mathrm{kcal})$ in non-obese Japanese patients with type 2 diabetes $(\bullet \bullet, n=23)$ or non-obese control subjects with normal glucose tolerance $(\circ \circ, n=13$ ) following 10 hours of overnight fasting. The mean of BMI was not significantly different between groups.

\section{AUC}

In the term of AUC, mean PG was significantly $(p<0.001)$ higher in group $1(681 \pm 26 \mathrm{mg} \mathrm{min} / \mathrm{dL})$ than in group $2(307 \pm 11 \mathrm{mg} \mathrm{min} /$ $\mathrm{dL})$. However, there were no significant differences in s-IRI $(50 \pm 7 \mu \mathrm{U}$ $\mathrm{min} / \mathrm{mL}$ versus $51 \pm 4 \mu \mathrm{U} \mathrm{min} / \mathrm{mL}$, respectively), s-CPR $(9.3 \pm 0.8 \mathrm{ng}$ $\mathrm{min} / \mathrm{mL}$ versus $9.2 \pm 0.5 \mathrm{ng} \mathrm{min} / \mathrm{mL}$, respectively) and $\mathrm{p}$-active GLP$1-\mathrm{S}(16.1 \pm 1.4 \mathrm{pmol} \mathrm{min} / \mathrm{L}$ versus $18.7 \pm 2.8 \mathrm{pmol} \mathrm{min} / \mathrm{L}$, respectively) levels between two groups.

\section{Discussion}

The patients were Japanese with T2DM, who had non-obesity with a tendency of insulin resistance based on the criteria of Matthews et al. [7] and low insulin secretion associated with low early-phase after TM, which was confirmed by higher HOMA-R, lower HOMA- $\beta$ values and lower insulinogenic index than by those in control group, although there were insignificant differences in means of s-IRI and s-CPR in fasting and postprandial levels after TM between both groups. As patients had long durations of diabetes with mean of 9.2 years, some patients had micro- and macro-vascular complications with various severities varied, and had various medicines without incretin-related drugs.

There were no significant differences in fasting and postprandial p-active GLP-1-S levels after TM between groups similar to the report of Lee et al. [3], and the peaked time point of s-active GLP-1-S after TM was also same in two studies. However, the peaked level (around $5 \mathrm{pmol} / \mathrm{L}$ ) in the study by Lee et al. [3] was slightly lower than that our study. The difference may be due to the differences of total caloric contents used as TM ( $480 \mathrm{kcal}$ versus $520-560 \mathrm{kcal}$ ). Recently, the Japan Diabetes Society and the Japan Association for Diabetes Education and Care Committee for Standardized Incretin Measurement recommends strongly that when p-active GLP-1 is measured, it should be measured the sample extracted by ethanol or other methods [8]. The reason is due to that foreign substances in unextracted samples might interfere in the assay [9]. However, p-active GLP-1 levels to response to ingestion of TM in unextracted samples measured by ELISA kit used in this study were correlated to p-total GLP-1 levels measured by RIA kit in extracted samples by ethanol [10]. Also, Yi et al. showed p-active GLP-1 level measured by this kit in unextracted sample by mass spectrometry analyzing was almost active GLP-1 [11]. Moreover, means of p-active GLP-1 levels measured by ELISA kit used in this study in extracted samples of non-obese Japanese patients with T2DM $(n=41)$ [9] were similar to those in the study with unextracted samples by Lee at al. [3], although the variation was higher in unextracted samples than in extracted samples [9].

Another difference between our findings and those of Lee et al. is the glycemic state. Mean HbAlc $(9.1 \pm 0.3 \%)$ (NGSP) in our patients was significantly $(p<0.001)$ higher than that $(6.8 \pm 0.5 \%)$ (NGSP) in patients by Lee et al. [3]. Hyperglycemia evaluated by HbA1c levels increases serum DPP-IV activity in patients with T2DM [11]. Moreover, serum DPP-IV activity is positively correlated to HbAlc levels in patients with T2DM [13]. Hence, the increased DDP-IV activity in the patients by this study should result in lower p-active GLP-1 levels. However, the findings in the patients were similar to those in the healthy individuals. Therefore, the glycemic state may not be directly related to these similar results.

The most difference between Lee et al. and our studies was the difference in demographic characteristics of patients participated. 
Citation: Kamoi K, Ohara N, Tomoo I, Shinozaki Y, Furukawa K (2011) Normal Response of Active GLP-1 like Substances Level to Test Meal in Non-Obese Type 2 Diabetic Japanese Patients with Complications and Receiving Treatments. J Diabetes Metab 2:147. doi:10.4172/21556156.1000147

The patients by Lee et al. were newly diagnosed as diabetes, had no complications and did not use medical drugs [3]. Therefore, patients by Lee et al. were complication- and treatment-naïve for T2DM, but our patients were not naïve. However, there was no statistical difference $(\mathrm{r}=0.002, p=0.820, \mathrm{n}=22)$ in association between the AUCs of $\mathrm{p}$-active GLP-1-S and the duration of diabetes. Yabe et al. [9] reported there was no significant difference in p-active GLP-1 levels with AUC following ingestion of TM between extracted samples of non-obese Japanese patients with T2DM and controls, whose durations of disease were similar to those in this study.

Further, SU or TZD did not influence the secretion of active GLP$1[9,14,15]$, while $\alpha$-GI or BG may enhance the secretion of active GLP- $1[16,17]$. Hence, the p-active GLP-1 levels in patients treated with $\alpha$-GI $(n=1)$ or BG $(n=15)$ in this study should result in higher than those in control. However, actual levels of p-active GLP-1-S were similar to those in the healthy individuals. The reason is not clarified, but some researchers reported therapy combined of a-GI or BG and DPP-IV inhibitors or GLP-1 agonist in patients with T2DM enhanced or increased plasma GLP-1 levels significantly [16,17]. Also, anti-hyperlipidemic or anti-hypertensive medicines do not influence secretion of active GLP-1.

In some diabetic complications, autonomic neuropathy may decrease incretin effect [18], whereas chronic renal failure (serum creatinine $2.2 \pm 0.9 \mathrm{mg} / \mathrm{dl}$ ) may result in delayed elimination of GLP1 secretin [19]. Although we did not precisely examine autonomic neuropathy as others laboratory tests as previously reported methods $[2,20]$, the patients did not complain the clinical symptoms such as orthotic hypotension, awareness hypoglycemia or other related disturbances. Autonomic neuropathy is related to duration of disease. However, Yabe at al. [9] reported there was no significant difference in p-active GLP-1 levels with AUC following ingestion of TM between extracted samples of non-obese Japanese patients with T2DM and controls, whose durations of disease were similar to those in this study. Also, Toft-Nielsen et al. reported there was no statistical association between p-active GLP-1 levels and autonomic neuropathy in patients with T2DM [20]. Further, although one patent in this study had macroalbuminuria, the serum creatinine levels were less than $1.0 \mathrm{mg} / \mathrm{dl}$ with more than $60 \mathrm{~mL} /$ minute $/ 1.73 \mathrm{~m} 2$ of GFR. Therefore, these factors do not influence p-active GLP-1-S levels in this study as indication by Toft-Nielsen et al. [20].

It is well known that the post prandial levels of the other incretin GIP are similar between T2DM and healthy individuals [2,3]. However, we did not measure postmeal GIP levels. It would be interesting in future study to evaluate the incretin effect in the subjects by measuring insulin or C peptide after 75g OGTT and after isoglycemic IV glucose infusion.

\section{Conclusion}

Our results indicated that basal and postprandial levels of plasma active GLP-1-S after a test meal in non-obese Japanese patients with T2DM associated with a long duration of the disease, various complications and various treatments except related-incretin drugs were similar to those in non-obese healthy individuals.

\section{Acknowledgments}

We are grateful to clinical nursing staffs, clinical laboratory technicians and clinical dietitians of Nagaoka Red Cross Hospital and Niigata Medical Center for their valuable technical assistances.

\section{References}

1. Kieffer TJ, Mclntosh CH, Pederson RA (1995) Degradation of glucosedependent insulinotropic polypeptide and truncated glucagon-like peptide 1 in vitro and in vivo by dipeptidyl peptidase IV. Endocrinology 136: 3585-3596.

2. Vilsbøll T, Krarup T, Sonne J, Madsbad S, Vølund A, et al. (2003) Incretin secretion in relation to meal size and body weight in healthy subjects and people with type 1 and type 2 diabetes mellitus. J Clin Endocrinol Metab 88 2706-2713.

3. Lee S, Yabe D, Nohtomi K, Takada M, Morita R, et al. (2009) Intact glucagonlike peptide- 1 levels are not decreased in Japanese patients with type 2 diabetes. Endocr J 57: 119-126.

4. Examination Committee of Criteria for 'Obesity Disease' in Japan; Japan Society for the Study of Obesity (2002) New criteria for 'obesity disease' in Japan. Circ J 66: 987-992.

5. Kamoi K, Shinozaki Y, Furukawa F, Sasaki H (2011) Decreased active GLP-1 response following large test meal in patients with type 1 diabetes using bolus insulin analogues. Endocrine $\mathrm{J}$.

6. World Health Organization (1999) Definition, diagnosis and classification of diabetes and its complications. Part 1: diagnosis and classification of diabetes mellitus. Geneva: Department of Noncommunicable Disease Surveillance.

7. Matthews DR, Hosker JP, Rudenski AS, Naylor BA, Treacher DF, et al. (1985) Homeostasis model assessment: insulin resistance and beta-cell function from fasting plasma glucose and insulin concentrations in man. Diabetologia 28 412-419.

8. The Japan Diabetes Society and the Japan Association for Diabetes Education and Care Committee for Standardized Incretin Measurement (2011). Guideline for Incretin Measurement.

9. Yabe D, Watanabe K, Sugawara K, Kuwata H, Kitamoto Y, et al. (2011) Comparison of incretin immunoassays with or without plasma extraction: incretin secretion in Japanese patients with type 2 diabetes. J Diabetes Invest.

10. Heijboer AC, Frans A, Lomecky M, Blankenstein MA (2011). Analysis of glucagon-like peptide 1; what to measure? Clin Chim Acta 412: 1191-1194.

11. Yi J, Liu Z, Warunek D, Song Y-Q, Khumush L, Craft D (2010). Ex vivo stabilization of GLP-1 and GIP in human plasma

12. Mannucci E, Pala L, Ciani S, Bardini G, Pezzatini A, et al. (2005) Hyperglycaemia increases dipeptidyl peptidase IV activity in diabetes mellitus. Diabetologia 48 1168-1172.

13. Ryskjaer J, Deacon CF, Carr RD, Krarup T, Madsbad S, et al. (2006) Plasma dipeptidyl peptidase-IV activity in patients with type-2 diabetes mellitus correlates positively with HbAlc levels, but is not acutely affected by food intake. Eur J Endocrinol 155: 485-493.

14. Fukase N, Manaka H, Sugiyama K, Takahashi H, Igarashi M, et al. (1995) Response of truncated glucagon-like peptide-1 and gastric inhibitory polypeptide to glucose ingestion in non-insulin dependent diabetes mellitus. Effect of sulfonylurea therapy. Acta Diabetol 32: 165-169.

15. Larsen PJ, Wulff EM, Gotfredsen CF, Brand CL, Sturis J, et al. (2008) Combination of the insulin sensitizer, pioglitazone, and the long-acting GLP-1 human analog, liraglutide, exerts potent synergistic glucose-lowering efficacy in severely diabetic ZDF rats. Diabetes Obes Metab 10: 301-311.

16. Göke B, Fuder H, Wieckhorst G, Theiss U, Stridde E, et al. (1995) Voglibose (AO-128) is an efficient alpha-glucosidase inhibitor and mobilizes the endogenous GLP-1 reserve. Digestion 56: 493-501.

17. Cuthbertson J, Patterson S, O'Harte FP, Bell PM (2011) Addition of metformin to exogenous glucagon-like peptide-1 results in increased serum glucagonlike peptide- 1 concentrations and greater glucose lowering in type 2 diabetes mellitus. Metabolism 60: 52-56.

18. Kazakos KA, Sarafidis PA, Yovos JG (2008) The impact of diabetic autonomic neuropathy on the incretin effect. Med Sci Monit 14: CR213-220.

19. Meier JJ, Nauck MA, Kranz D, Holst JJ, Deacon CF, et al. (2004) Secretion degradation, and elimination of glucagon-like peptide 1 and gastric inhibitory polypeptide in patients with chronic renal insufficiency and healthy control subjects. Diabetes 53: 654-662.

20. Toft-Nielsen MB, Damho TMB, Madsbad S, Hilsted LM, Hughes TE, et al (2001) Determinants of impaired secretion of glucagonlike peptide-1 in type 2 diabeti patients. J Clin Endocrinol Metab 86: 3717-3723. 